\title{
Bioanalysis
}

\section{An ex vivo potency assay to assess active drug levels of a GLP-1 agonistic peptide during preclinical safety studies}

Background: During development of biologics, safety and efficacy assessments are often hampered by immune responses to the treatment. To assess active exposure of a drug peptide in a toxicology study, we developed an ex vivo potency assay which complemented the total drug quantification assay. Methodology: Compound activity was assessed in samples of treated monkeys by cell-based CAMP measurements. For each animal, activity was compared with its predose sample to which the compound has been added at the postdose concentration as determined by a total LC-MS/MS assay. Conclusion: We were able to show that despite a high total test compound level, activity was reduced tremendously in antidrug-antibody-positive monkeys. Therefore, the applied ex vivo potency assay supplements drug quantification methods to determine active exposures.

\section{Background}

During in vivo assessments of biologics including therapeutic peptides, it is important to correlate the amount of drug in circulation with safety and efficacy parameters in individual animals. This correlation is often hampered by the occurrence of circulating antidrug antibodies (ADAs), which may or may not affect both the PK and the PD of the drug.

Induction of an immune response to biologics/therapeutic peptides may not occur on the first treatment cycle, but is commonly observed after multiple doses. Even biologics that are produced as recombinant copies of endogenous molecules may induce ADA formation, thereby profoundly affecting efficacy and safety [1]. ADAs might affect the relationship between the dose applied, the drug concentration achieved in plasma and tissue and the PD effects of treatment [1]. Furthermore, ADAs either increase or decrease the clearance rate of therapeutic monoclonal antibodies [2] and therapeutic peptides [3]. However, the sheer presence of ADAs does not allow any conclusions on their impact on the safety and efficacy of biologics/therapeutic peptides. After long-term treatment in chronic toxicity studies, the majority of experimental animals might develop an immune response, with a high variability in ADA titers and $\mathrm{PK} / \mathrm{PD}$ consequences among individual animals $[3,4]$. The questions whether and to what extent ADA-bound biologics retain pharmacological activity is, therefore, crucial for the interpretation of efficacy and safety data.

Bioanalytical methods have been reported which are either used for the quantification of the total drug concentration or of a fraction of the drug, such as 'active' or 'targetbinding competent drug' [5]. It is dependent on the nature of the drug, on the desired read-out of the study and on the technical possibilities which method is used for drug quantification.

In case a total drug quantification is used for a certain study, assays to measure the amount of its biological activity may be needed to complement the assay battery for $\mathrm{PK} / \mathrm{PD}$ assessment in chronic toxicity studies.

Such a case is a therapeutic peptide acting as agonist of the GLP-1, hereafter referred to as 'test compound'. This test compound acts as an incretin-mimicking peptide intended to exert antihyperglycemic and insulino-
Martin Schäfer ${ }^{*, 1}$, Steven Challand', Eginhard Schick², Sabine Bader ${ }^{1}$, Dominik Hainzl $^{3}$, Katja Heinig ${ }^{2}$, Lutz Müller ${ }^{2}$, Apollon Papadimitriou ${ }^{1}$ \& Julia Heinrich ${ }^{1}$

'Roche Pharmaceutical Research \& Early Development, Pharmaceutical Sciences, Roche Innovation Center Penzberg, Roche Diagnostics $\mathrm{GmbH}$, Nonnenwald 2, 82377 Penzberg, Germany

${ }^{2}$ Roche Pharmaceutical Research \& Early Development, Pharmaceutical Sciences, Roche Innovation Center Basel, F. Hoffmann-La Roche Ltd, Grenzacherstrasse 124, 4070 Basel, Switzerland

${ }^{3}$ Metabolism \& Pharmacokinetics, Novartis Institute for BioMedical Research, 250 Massachusetts Ave, Cambridge, MA 02139, USA *Author for correspondence: Tel.: +4988566019099 Fax: +49 8856607919099 martin.schaefer@roche.com 


\section{Key terms}

LC-MS/MS: An analytical technology for separation, quantification and potential identification of drugs and metabolites of particular masses.

Ex vivo potency assay: A cell-based assay reflecting the mode of action of a biotherapeutic used to assess its ex vivo activity.

GLP-1 receptors: In pancreatic $\beta$ cells, activated GLP-1R stimulates the CAMP pathway, resulting in an increased synthesis and release of insulin. In the brain, GLP-1R is involved in the control of appetite.

Bridging ELISA: The bridging ELISA bases on the two antigen-binding sites of antibodies, thus allowing a bridge to form between the drug immobilized on plastic wells and the labeled drug that is added in the detection step.

tropic effects that might be beneficial for the chronic treatment of patients with diabetes and obesity [6]. Preclinical safety studies in rodents and nonrodents, therefore, require long treatment durations of up to 26 and 39 weeks, respectively [7].

Exaggerated PD effects, which are particularly pronounced in nondisease animal models, have been observed for other incretin-mimicking peptides during toxicity studies [8]. These effects include a marked decrease of blood glucose and food intake, especially in lean animals on restricted diet like those used in toxicology studies. Moreover, GLP-1 analogs such as exenatide and albiglutide are known to induce ADA formation, whose titers are highly variable among experimental animals in toxicity studies [3,4]. This may be the reason why a high variability in total drug exposure and, thereby, PD effect was observed in animals treated with albiglutide at the same dose level [4]. Due to the observed heterogeneity in immune responses and the fact that PD effects did not correlate to dose, apparent exposure and the occurrence of ADA, additional assays to assess the biological activity of incretinmimicking therapeutic peptides seemed to be required in chronic toxicity studies to assess whether animals are still exposed to active drug despite the presence of ADAs.

In our case, total drug levels were quantified during a toxicity study by using liquid chromatographytandem mass spectrometry (LC-MS/MS [8]). Sample preparation in this method interrupts interactions between therapeutic peptides and ADA or plasma proteins. Therefore, LC-MS/MS cannot differentiate between total and active peptide levels [9,10]. From a physiological point of view, cell-based assays that use readout systems based on the cellular mode of action would be the most relevant system to assess the amount of active biotherapeutics. Such assays have successfully been applied to quantify the active frac- tion of biologics such as human monoclonal antibodies to human HGF [11] and recombinant proteins with erythropoiesis stimulating properties [12].

Here, we report the development of a cell-based ex vivo potency assay to assess the activity of a peptide. This assay is based on the induction of intracellular cAMP synthesis by activated GLP-1 receptors. To assess the amount of active test compound, both activity (by the ex vivo potency assay) and total concentration (by LC-MS/MS) of the test compound are measured in plasma samples from experimental animals after administration of the test compound (postdose sample). Then, the naive predose plasma samples of the identical, untreated animals are spiked with the test compound at equimolar concentrations. The relative comparison of the activity in post- and pre-dose samples then allows assessing the impact of an individual immune response, as well as other biological processes, on drug activity. Plasma samples used during assay development and qualification were obtained from a previous developmental study analyzing the effects of the test compound in cynomolgus monkeys.

\section{Methods}

Test compound

The test compound is a synthetic peptide. The sample batch used for all experiments had a purity greater than $95 \%$. For all experiments, the test compound was dissolved at 224 and $1120 \mu \mathrm{M}$ in $20 \mathrm{mM}$ histidine/histidine- $\mathrm{HCl}$ buffer, $230 \mathrm{mM}$ trehalose, $10 \mathrm{mM}$ methionine, $0.04 \%$ polysorbate 20 ( $\mathrm{pH} 7.0$ ). The stability of the test compound in the final solution was analytically confirmed by reversed-phase ultra-performance liquid chromatography (RP-UPLC).

\section{Animal procedures}

Plasma samples from cynomolgus monkeys were obtained during a 39-week toxicity and toxicokinetic study that was conducted during drug development. In this study, animals received a subcutaneous once daily injection of the test compound at $0.015,0.15$ and $0.1 \mathrm{mg} / \mathrm{kg} /$ day. Control animals received vehicle control only.

Four animals per dose and sex were treated during the 39-week study. On day 1, 28, 85, 175 and 267, plasma samples were taken before and 1, 3, 7 and $24 \mathrm{~h}$ after administration of the test compound. ADA levels were assessed at week 1, 39, 41, 43 and 47. The animal study referred to in this report was conducted in compliance with good laboratory practice and the applicable OECD and ICH guidelines for chronic nonrodent toxicity studies, animal permits and applicable animal regulations [13,14]. 


\section{LC-MS/MS}

To measure total concentrations of the test compound in plasma samples, LC-MS/MS was performed as described [9]. In brief, this method involves a columnswitching liquid chromatography followed by tandem mass spectrometric detection. The calibration range was 2.00 to $1000 \mathrm{ng} / \mathrm{ml}$ of the test compound in $50 \mu \mathrm{l}$ aliquots of K3-EDTA cynomolgus monkey plasma. For sample preparation, proteins were precipitated from plasma samples $(50 \mu \mathrm{l})$ by addition of ethanol $(50 \mu \mathrm{l})$ and acetonitrile $(200 \mu \mathrm{l})$ to dissolve complexes of other proteins with the peptide. Stable isotope labeled test compound $\left({ }^{13} \mathrm{C}_{9}{ }^{15} \mathrm{~N}\right)$ served as internal standard. For mass spectrometric detection, we used selected reaction monitoring (SRM) in the positive ion mode (AB Sciex API5000 triple-quadrupole mass spectrometer). The selected ion reactions $(\mathrm{m} / \mathrm{z})$ were $1119.35(\mathrm{M}+4)^{4+} \rightarrow 762.55$ for the peptide drug and $1121.85(\mathrm{M}+4)^{4+} \rightarrow 762.55$ for the internal standard. The method to measure total concentrations of the test compound in plasma samples was fully validated [Guenzi A, Unpublished Data] and met the acceptance criteria set forth by regulatory agencies $[15,16]$.

\section{ADA bridging ELISA}

For the qualitative detection of antibodies directed against the test compound, a sandwich bridging ELISA was used. Test samples contained 10\% (v/v) plasma. An immunoaffinity purified antibody directed against AA 1-12 of the test compound (Roche Diagnostics $\mathrm{GmbH}$ ) was used to prepare positive quality control (QC) samples. Test and QC-samples were incubated overnight $(16-18 \mathrm{~h})$ at room temperature (RT) on an MTP shaker (500 rpm), in the presence of the biotinylated and digoxigenylated test compound (Roche Diagnostics $\mathrm{GmbH}$ ) as capture and detection reagent, respectively. Immune complexes were then transferred to a streptavidin-coated microtiterplate and incubated for $1 \mathrm{~h}$ (RT) to immobilize immune complexes. The supernatant was aspired, and unbound substances were removed by subsequent washing steps in phosphate buffered saline (PBS) containing 0.05\% n-octyl glucoside (Roche Diagnostics $\mathrm{GmbH}$ ). Samples were then incubated with $7 \mathrm{mU}$ horseradish peroxidase conjugated anti-digoxigenin Fab fragments (Roche Diagnostics $\mathrm{GmbH}$ ) for $1 \mathrm{~h}$ at RT, washed and incubated for $45 \mathrm{~min}$ at RT in 3,3',5, 5'-tetramethylbenzidine (TMB) substrate solution (Roche Diagnostics $\mathrm{GmbH})$. Sulfuric acid was added to stop the reaction, and color intensity was determined photometrically at $490 \mathrm{~nm}$ (650 $\mathrm{nm}$ reference wavelength).

In a screening assay, plasma samples were tested for the presence of antitest compound antibodies at a false-positive rate of $5 \%$. For positive controls, the assay had a sensitivity of $5.61 \mathrm{ng} / \mathrm{ml}$ in $100 \%$ plasma. Positive results were confirmed in a second, confirmatory assay which was identical to the screening assay with the exception that test samples were preincubated with or without an excess of the test compound $(250 \mathrm{ng} / \mathrm{ml}$ final assay concentration). A sample was defined as ADA positive if the percentage of signal quenching was $\geq 13.5 \%$ in the presence of the spiked test compound as calculated during assay validation following the respective recommendations $[17,18]$. The method to detect antibodies directed against the test compound was fully validated [Araya M, Unpublished Data] according to the standard of the pharmaceutical industry $[17,18]$.

\section{Ex vivo potency assay}

To assess the amount of active test compound, both activity, as assessed by cAMP measurement, and amount of total test compound, as assessed by LC-MS/MS, were analyzed in plasma samples from experimental animals after administration of the test compound (postdose sample). For each individual animal, the activity of the postdose sample was compared with that of the naive predose plasma sample to which the test compound had been added at the concentration quantified by the total LC-MS/MS assay in the postdose sample.

The whole ex vivo potency assay procedure comprised three experimental steps. First, at day 1, an assay plate was prepared by seeding 5000 stably GLP-1R expressing CHO-K1 cells (DiscoveRx; product code 95-0062C2) per well in a black 96-well plate, followed by overnight incubation at $37^{\circ} \mathrm{C}$. Second, at day 2, a 96-well template plate was prepared with cAMP standards, peptide calibrators, QC samples and preand post-dose samples ( $5 \%$ cynomolgus plasma, final concentration). Predose samples were spiked with the test compound at the total concentration found by LC-MS/MS quantification in the postdose sample of the identical animal, and both samples were diluted to obtain a final concentration of the test compound of 2 $\mathrm{nM}$. The template plate was preincubated at RT for 30 min. Standards were analyzed in duplicates, and quality controls as well as test samples were analyzed in triplicates. Then, the culture medium was removed from the assay plate and replaced with template samples $(50 \mu \mathrm{l})$. After a 30-min incubation on a Heidolph titramax 100 reciprocal shaker (500 rpm; RT), d2-labeled cAMP and anti-cAMP cryptate were added ( $25 \mu \mathrm{l} /$ well each), and the assay plate was incubated in the dark for $1 \mathrm{~h}$ at RT (reciprocal shaker at $500 \mathrm{rpm}$ ). Third, cellular cAMP levels were subsequently measured by the cAMP dynamic 2 kit (Cisbio; 62AM4PEC), according to the manufacturer's instructions [19]. Since free cAMP produced by CHO-K1 cells competes with cAMP-d2 for binding to anti-cAMP cryptate, fluorescence measure- 
ments (620 and $665 \mathrm{nM}$; Tecan infinite F500 reader) indicate the concentrations of intracellular cAMP.

\section{Statistical analysis}

A lower threshold for the test compound activity was defined as the $5 \%$ quantiles of differences (nM, cAMP) between matching 'postdose' and 'predose' samples. As the difference values were normally distributed, this threshold was calculated by a parametric quantile estimation (cut point $[\mathrm{CP}]=$ mean $[\mathrm{d}]-1.96 \times \mathrm{SD}[\mathrm{d}]$, with $\mathrm{d}=$ vector of differences, mean $[\mathrm{d}]=$ mean of the differences, $\mathrm{SD}[\mathrm{d}]=$ standard deviation of the differences). The variation from measurement to measurement was described by a statistical model based on Bayesian estimates. The resulting variance component information was considered to identify experimental factors that explain large amounts of the total variance. However, only a small amount of the total variance was explained by the factors sample (7\%), peptide concentration (7\%) and run (14\%). Therefore, all data points were pooled for the determination of the cut point value. The cut point was estimated as the empirical 5\% quantile of cAMP differences between pre- and post-dose samples [nM] across peptide concentrations, runs and samples. All statistical calculations and plots were performed using the software JMP 10.0.0 (SAS Institute Inc., 2012).

\section{Results}

\section{Assay development}

The ex vivo potency assay basically comprises three components, stably GLP-1R expressing CHO-K1 cells, a template plate containing test samples and standards and a competitive cAMP immunoassay as readout system. During assay development, we initially tried to calculate the amount of the active test compound from a cAMP calibration curve obtained from commercially available cynomolgus pooled plasma samples (CPP; firma) spiked with the test compound. In this experiment, amounts between 1 to $4 \mathrm{nM}$ test compound in CPP could be recovered within a range of $\pm 20 \%$ of the expected values. However, in five different experiments assessing the interindividual variability with plasma samples of six individual experimental animals, the signals of 1-4 nM test compound were out of this range in approximately $50 \%$ of animals when compared with the respective CPP samples (data not shown). Biological variability between individual animals is a typical phenomenon observed in cell-based activity assays that might be caused by different factors such as different cytokine levels between animals affecting cellular activity independent of the test compound.

We, therefore, decided to normalize the intra-animal data by assessing the activity of the test compound by comparing pre- and post-dose samples of individual animals. Concentrations of the test compound in postdose samples were assessed by LC-MS/MS, and predose samples of the identical animals were then spiked with the test compound at equimolar concentrations. Before measurement, samples were diluted to $2 \mathrm{nM}$ test compound. The comparison of the test compound activity in post- and pre-dose samples then indicated the change of test compound activity in the postdose sample (Figure 1).

\section{Determination of the cut point}

We determined a cut point of cAMP reduction that indicates a significant loss of compound activity. Six naive individual cynomolgus monkey plasma samples

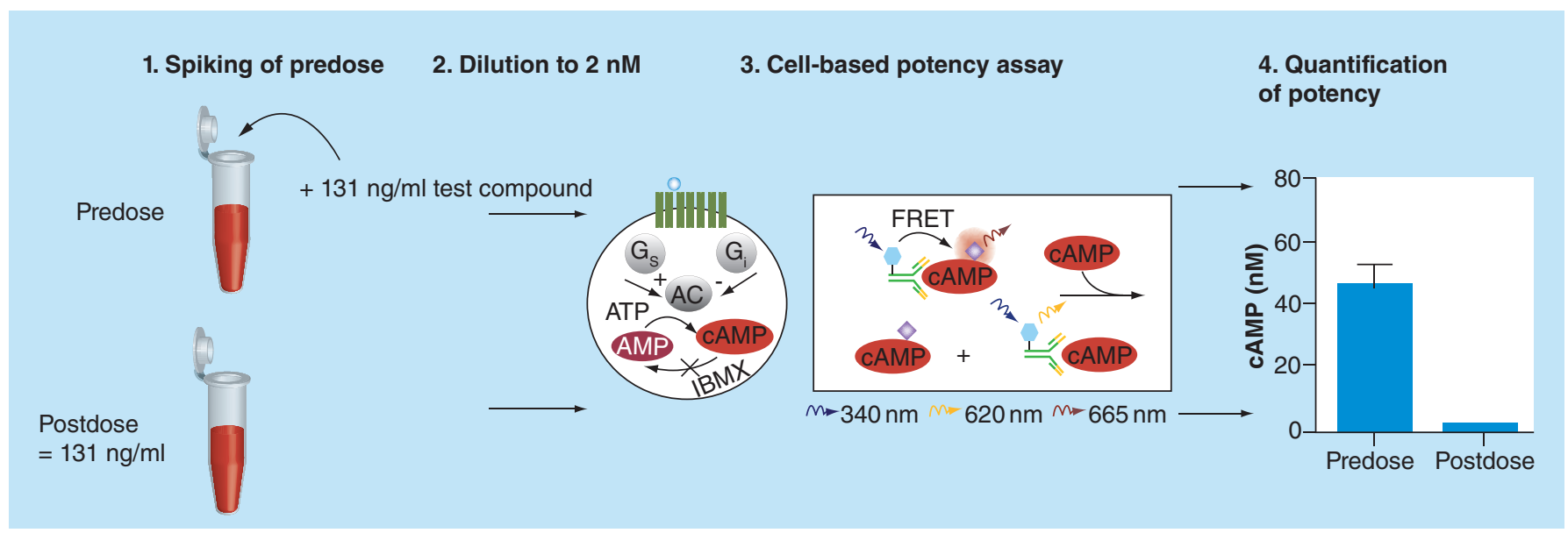

Figure 1. The ex vivo potency assay comprises four basic steps. First, a naive plasma sample (predose) is spiked with the test compound at the concentration found in the plasma of the identical animal after administration of the test compound (postdose; e.g., $131 \mathrm{ng} / \mathrm{ml}$ ). Second, both samples are diluted to obtain a final concentration of the test compound of $2 \mathrm{nM}$. Third, cellular cAMP levels are measured and, fourth, quantified by fluorescence measurements. The comparison of test compound activity in both samples then allows assessing the impact of immune responses and other biological processes on drug activity.

FRET: Fluorescence resonance energy transfer; IBMX: Isobutylmethylxanthine. 


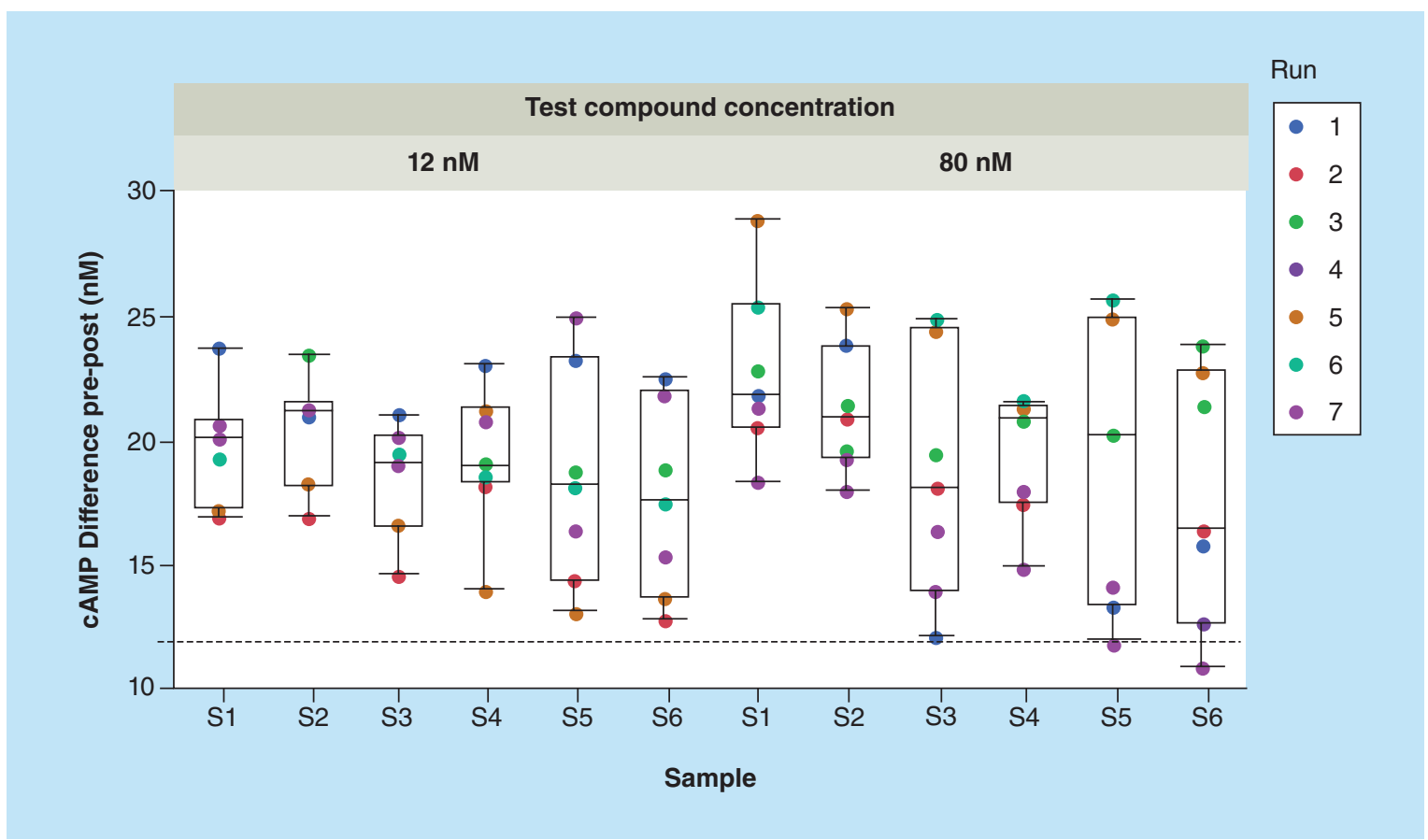

Figure 2. Statistical determination of the cut point of the ex vivo potency assay. Six naive cynomolgus monkey plasma samples (S1-S6) were spiked with the test compound at 12 or $80 \mathrm{nM}$, and an up to threefold molar excess of a polyclonal antitest compound antibody. Cut-point determination (dotted line) based on the pre- to post-difference in CAMP levels measured in seven experiments (run 1-7).

(predose samples) were spiked with 12 and $80 \mathrm{nM}$ test compound (postdose samples). Postdose samples additionally contained the 2.4-3.0-fold molar excess of a rabbit polyclonal positive control antibody against the test compound. These samples were then analyzed by the ex vivo potency assay in seven independent experiments. Statistical analyses defined the cut point as 11.9 nM cAMP difference between pre- and post-dose samples, and, accordingly a threshold for reduced test compound activity equivalent to a reduction by more than $12 \mathrm{nM}$ cAMP in postdose samples (Figure 2).

\section{Animal study}

Next, we employed our assay along with LC-MS/MS measurements and ADA bridging ELISA to characterize individual cynomolgus monkey plasma samples. LC-MS/MS measurements revealed steady-state total plasma concentrations of the test compound after 85 days of daily treatment. Accumulation ratios were approximately threefold or less. Substantially higher concentrations ( $\geq$ threefold $S D$ of the group mean after exclusion of outliers) were observed in 9 out of 36 animals. ADAs were detected not only in these nine animals with substantially higher test compound concentrations but in 32 out of 36 animals treated with the test compound. Analysis of plasma samples at day 267 revealed a general increase of the test compound plasma concentrations when compared with day 28 and, moreover, a reduced test compound clearance rate in individual animals such as \#3001 (Figure 3).

Based on these data, animals were allocated into three categories. In contrast to animals of category 1 , which were ADA negative, animals of the categories 2 and 3 were tested positive for ADA. Animals of the category 2 were further characterized by a markedly reduced total test compound clearance rate at day 267 , whereas animals of the categories 1 and 3 had normal clearance rates. Figure 4 exemplarily shows that test compound activity, as assessed by the ex vivo potency assay, was retained in individual ADA-positive as well as ADA-negative animals (category 1 and 3), while test compound activity was dramatically decreased in ADA-positive animals with a reduced test compound clearance rate at day 267 (\#3001 and \#2502).

In order to elucidate the time course of loss of activity, we analyzed additional postdose samples (day 8 to day 252) of individuals \#3001 and \#2502 in the ex vivo potency assay. We observed a continuous decrease of test compound activity in both animals, which was pronounced at day 85 . While the values of animal \#2502 remained stable at low activity levels, values of animal \#3001 dropped to zero, indicating a complete loss of test compound activity (Figure 5).

Interestingly, this loss of test compound activity in animal \#3001 coincided with the loss of efficacy of the test compound on bodyweight (PD marker). The effect on 

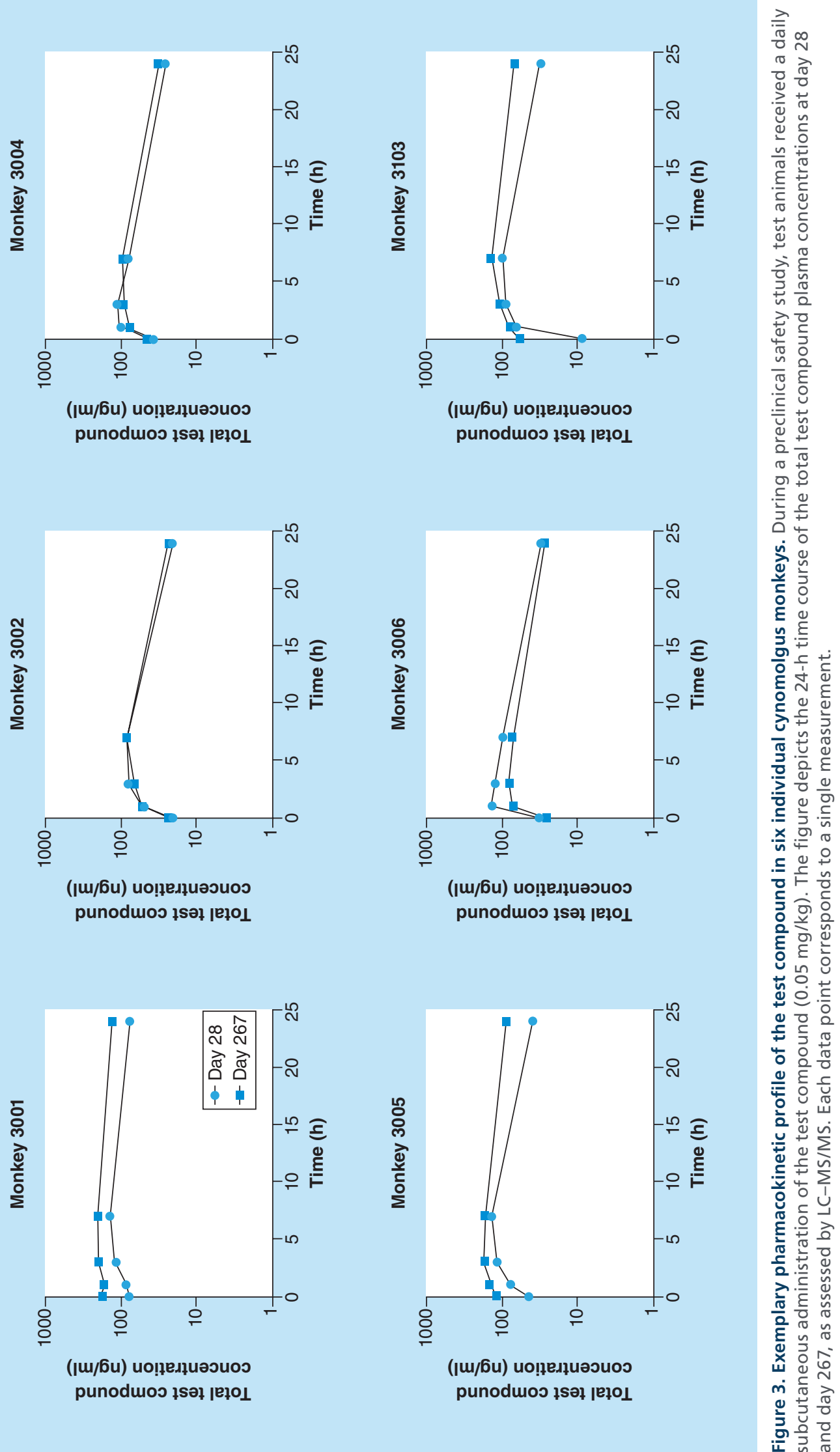

punodwos 골 ן라이 
bodyweight was not observed in animal \#2502, in which the test compound retained low but measurable activity (Figure 6). However, it might be worth noting that loss of bodyweight reduction did not generally indicate lack of drug activity in treated animals and is, therefore, a weak marker for PD activity (data not shown).

\section{Discussion}

Here, we report the development of an ex vivo potency assay to evaluate the pharmacological activity of a therapeutic peptide during long-term safety studies. This was necessary as only total drug levels alongside with a qualitative assessment of ADA-positivity was available and PD markers showed high variation between individual animals; therefore, an additional method to assess active drug exposure was needed to help in the interpretation of study results.

The ex vivo potency assay qualitatively evaluates the activity of the test compound in the plasma of experimental animals. This assessment bases on the comparison of drug activity detected in plasma of test compound treated animals with that detected in naive plasma samples of the identical animals, to which an equimolar concentration of the test compound was added. We defined a cut point of test compound activity as a significant reduction of activity being more than $12 \mathrm{nM}$ cAMP in postdose samples. Initially, we tried to quantify test compound activity by using a cAMP calibration curve obtained by known test compound concentrations added to commercially available individual and pooled plasma samples which would allow a precise quantification of active drug levels. However, data obtained from experimental animals revealed a considerable interassay and intersubject variability, suggesting matrix effects that affect reproducibility and might lead to inaccurate quantification [20]. We therefore believe that an assay based on a qualitative pre- and post-dose comparison in a single assay provides a more robust option to assess test compound activity. Combined with PD, total drug level and ADA results in long-term safety studies, this qualitative assay proofed to be valuable for the interpretation of our study.

Quantification of total circulating therapeutic peptide levels by LC-MS/MS and the subsequent correlation to efficacy or toxicology markers suffers from the inability to differentiate between active and inactive species which might result from peptide-ADA complexes formed during chronic toxicology studies. For the assessment of safety margins, it is of utmost importance to judge which concentration of circulating drug has an impact on safety and whether ADAs influence this interpretation since they have an impact on the level of active drug. As no quantitative PK assay for active drug levels was available for our compound,

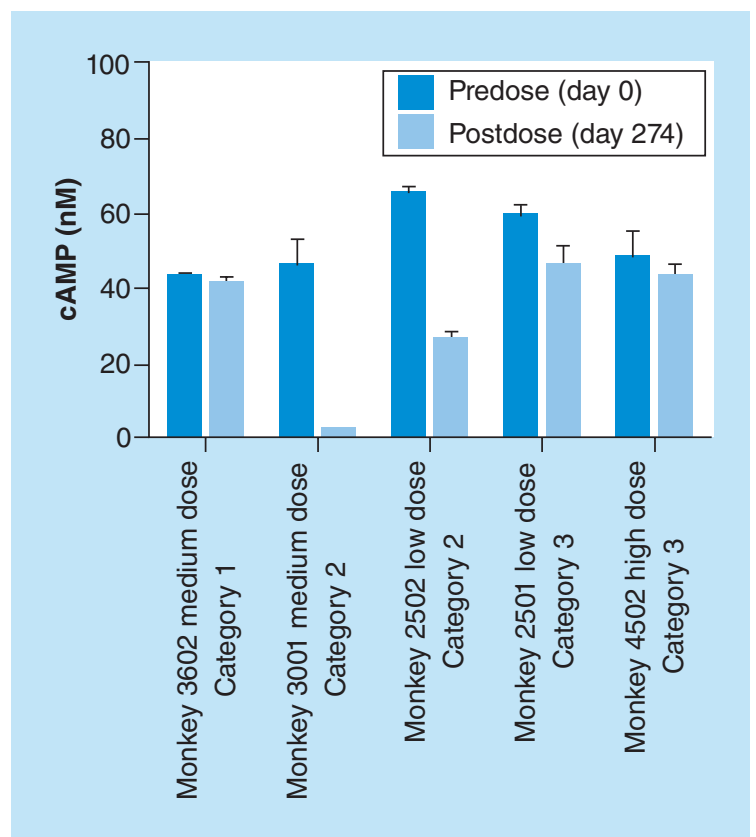

Figure 4. Analysis of test compound activity in individual cynomolgus monkeys. During a preclinical safety study, test animals received a subcutaneous once daily injection of the test compound at $0.015,0.15$ and $0.1 \mathrm{mg} / \mathrm{kg} /$ day (low, medium, high dose). Animals were categorized according to their antidrug-antibody (ADA) level (negative: category 1; positive: category $2,3)$ and test compound clearance rate at day 274 (normal: category 1, 3; reduced: category 2). Test compound activity of predose (day 0 , dark blue bars) and postdose samples (day 274, light blue bars) was assessed by the ex vivo potency assay, and cAMP levels were back-calculated from internal standard samples. Values are means \pm SD of triplicate measurements. a cell-based potency assay during chronic toxicology studies proved to be our method of choice for this assessment. Our data confirmed that the sheer presence of ADAs does not allow a conclusion on the exposure or activity of therapeutic peptides since it has been shown for this molecule class that ADAs can lead to a prolongation of half-life. Due to the fact that peptide-ADA complexes are often less effectively cleared from systemic circulation than unbound peptides, the overall drug exposure during long-term, multiple dose studies can be increased and lead to exaggerated PD effects when accompanied by an immune response [3].

In our chronic toxicity study in cynomolgus animals, we could indeed show that test compound activity was differentially affected by ADAs in individual monkeys. In one ADA-positive animal (\#3001), a complete loss of test compound activity occurred over time although total drug levels were higher as compared with other animals. Moreover, the time when the remaining activity decreased below $10 \%$ of the starting activity coincided with the onset of a fading effect on drug-induced bodyweight reduction. 


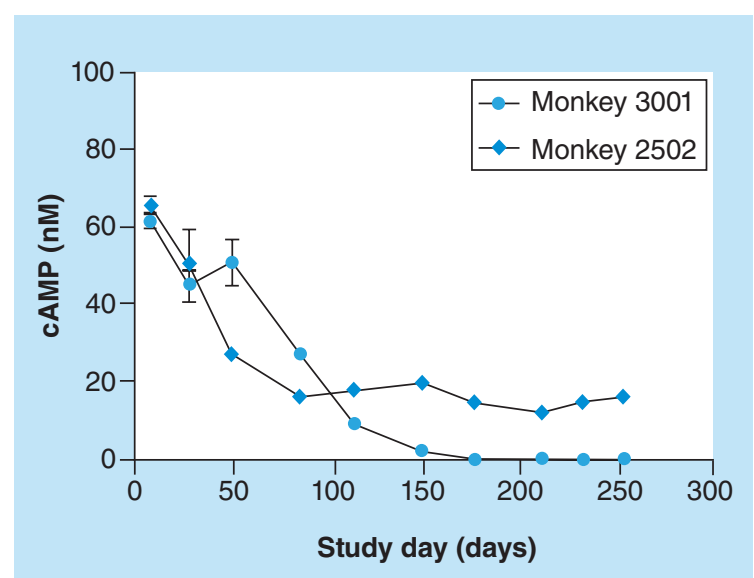

Figure 5. Time course of test compound activity in cynomolgus monkeys. Postdose plasma samples of two individual animals (\#3001 circles; \#2502 diamond) were obtained between day 8 and day 252 of a preclinical safety study. Samples were analyzed by the ex vivo potency assay, and cAMP levels were back-calculated from internal standards. Values are means \pm SD of triplicate measurements.

In other ADA-positive animals, however, the ADA effect on test compound activity ranged from 'not' or 'slightly' to 'severely affected' in the cell-based ex vivo potency assay. In one ADA-positive animal, where test compound activity was reduced to still measurable levels, the drug-induced effect on bodyweight could still be observed (\#2502).

These variable impacts of ADAs on test compound activity can be explained by different factors such as the epitope recognized by ADAs as well as overall ADA titers and affinity. Both high and low-affinity
ADAs might affect clearance and exposure, whereas only high-affinity ADAs might completely abolish drug activity and low-affinity ADAs might dissociate from the drug making it still able to activate their target receptors. However, even high-affinity ADA binding to distinct epitopes lying outside the receptorinteraction domain of the test compound may still allow pharmacological activity. Therefore, these considerations, along with our experimental observations, underscore the relevance of ex vivo potency assays for safety evaluations in preclinical toxicology studies.

We recognized two limitations of our assay. First, our assay does not allow precise quantification of test compound activity, a limitation that typically also applies to other cell-based bioanalytical assays. Second, it might be challenging to adapt this cell-based assay to a high-throughput format requiring outsourcing to external laboratories. This underscores the value and necessity of PK assays quantifying the active rather than total drug levels which are based on assay formats allowing for a high sample throughput [21]. Therefore, a well-defined bioanalytical assay strategy is needed a priori, especially for late-stage development.

\section{Conclusion}

By applying a cell-based ex vivo potency assay, we were able to show that despite a high total test compound level, implicating active drug exposure, drug activity was reduced tremendously in ADA-positive monkeys. Therefore, the applied ex vivo potency assay complements total drug quantification methods to determine meaningful exposures in preclinical safety studies.

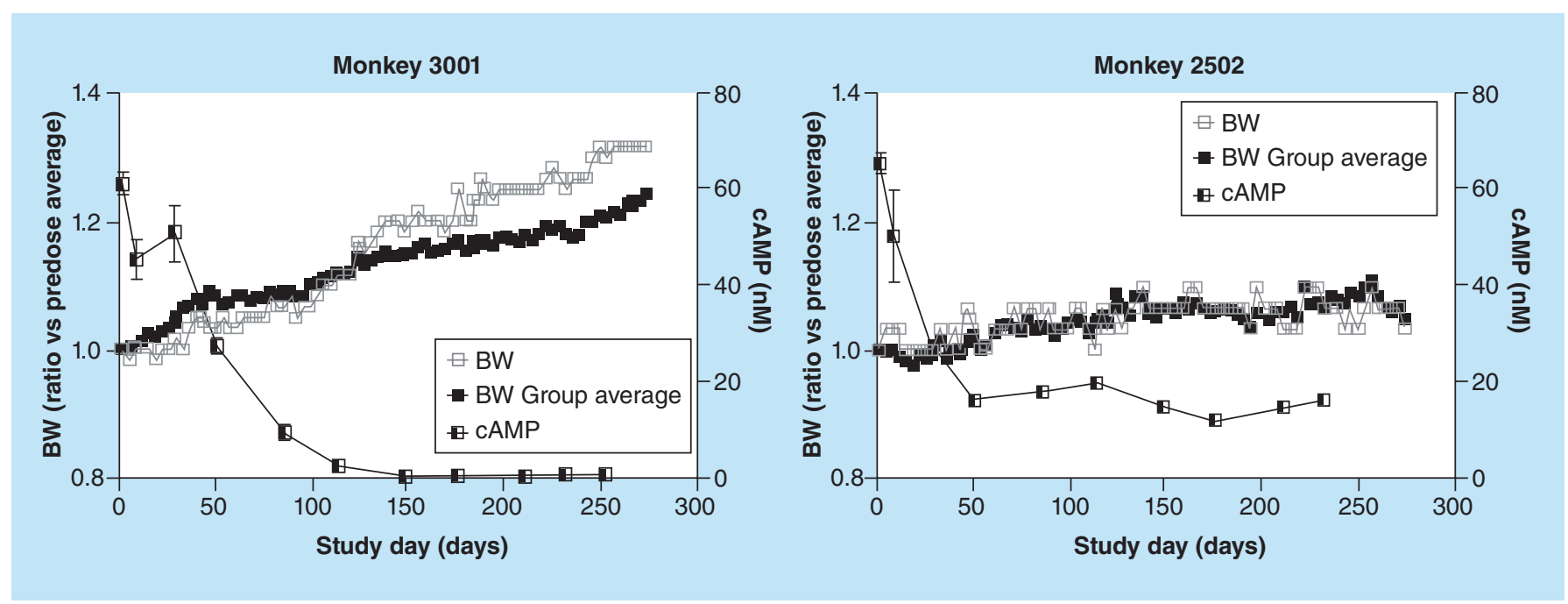

Figure 6. Correlation of test compound activity and pharmacodynamics effects. Test compound activity, as assessed by the ex vivo potency assay, and BW were evaluated in two individual animals (left: \#3001; right: \#2502) between day 8 and day 252 of a preclinical safety study. Values of cAMP levels (right y-axis) are means \pm SD of triplicate measurements. BW changes are presented as ratio of post- to pre-dose values (gray open square). For comparison, the average of BW changes in the corresponding group of animals is shown (black filled square).

BW: Bodyweight. 


\section{Future perspective}

Over the last couple of years the value of cell-based assays such as neutralizing antibody assays or cellbased PK methods has been widely discussed to assess drug activity in a physiological system. Here we show the successful implementation of a cell-based ex vivo potency assay that offered significantly improved information on drug activity in vivo. Since quantification of total drug levels might overestimate the amount of active drug exposure, the further evolution of methods to quantify active drug as complementary tool to total quantification methods or as stand-alone technique will get more importance in the future. This is especially the case for peptide drugs, for which total quantification by LC-MS/MS methods is currently typically established. However, as cell-based assays in many cases lack the possibility of precise quantification, high-throughput capability and suffer from sensitivity to drug-unrelated matrix effects, further alternative noncell-based assay formats (e.g., ligandbinding assays for active drug quantification) need to be investigated and implemented as key component of the assay battery for PK/PD assessment.

\section{Acknowledgements}

The authors would like to thank Michael Otteneder for fruitful discussions on the project.
Financial \& competing interests disclosure

M Schaefer, S Challand, S Bader, A Papadimitriou and J Heinrich are employed by Roche Diagnostics GmbH. K Heinig, E Schick and L Mueller are employed by F. Hoffmann-La Roche Ltd. D Hainzl is employed by Novartis and was employed by F. Hoffmann-La Roche Ltd at the time of the study. Some authors are owners of Roche bonus shares and profit certificates. The authors have no other relevant affiliations or financial involvement with any organization or entity with a financial interest in or financial conflict with the subject matter or materials discussed in the manuscript apart from those disclosed.

Writing assistance was provided by Stefan Lang, funded by Roche Diagnostics $\mathrm{GmbH}$.

\section{Ethical conduct of research}

The authors state that they have obtained appropriate institutional review board approval or have followed the principles outlined in the Declaration of Helsinki for all human or animal experimental investigations. In addition, for investigations involving human subjects, informed consent has been obtained from the participants involved.

\section{Open access}

This work is licensed under the Attribution-NonCommercialNoDerivatives 4.0 Unported License. To view a copy of this license, visit http://creativecommons.org/licenses/by-nc$\mathrm{nd} / 4.0 /$

\section{Executive summary}

- We developed a cell-based ex vivo potency assay to evaluate the pharmacological activity of an incretin mimicking therapeutic peptide in vivo.

- In this assay, plasma samples were directly analyzed in a cell-based readout system in which peptide activity in the unknown study sample is directly compared with a spiked predose sample of the same animal.

- By analyzing plasma samples of a safety study in cynomolgus monkeys, we found that reduction of peptide activity cannot be concluded from total drug levels, the sheer presence of ADA and from clinical parameters since ADAs had a heterogeneous effect on drug activity in individual animals.

- Therefore, the ex vivo potency assay complemented the methods for PK/PD assessment in a chronic toxicity study, with the goal to understand active drug exposure.

\section{References}

Papers of special note have been highlighted as: $\bullet$ of interest

1 Chirmule N, Jawa V, Meibohm B. Immunogenicity to therapeutic proteins: impact on $\mathrm{PK} / \mathrm{PD}$ and efficacy. AAPS J. 14(2), 296-302 (2012).

- Reviews the diversity of a potential impact caused by immunogenicity to therapeutic proteins.

2 Lecluse LA, Driessen RB, Spuls PI. Extent and clinical consequences of antibody formation against adalimumab in patients with plaque psoriasis. Arch. Dermatol. 146(2), 127-132 (2010).

3 Food and Drug Administration, Center for Drug Evaluation and Research. Pharmacology Review (21-919). www.accessdata.fda.gov
$4 \quad$ Mc Govern TJ, Food and Drug Administration, Center for Drug Evaluation and Research. Pharmacology Review (125431Orig1s000). www.accessdata.fda.gov

- Illustrates the high variability of immune responses and its resulting effect on PK in case of albiglutide-treated animals.

5 Staack RF, Jordan G, Dahl U et al. Free analyte QC concept: a novel approach to prove correct quantification of free therapeutic protein drug/biomarker concentrations. Bioanalysis 6(4), 485-496 (2014).

6 Wideman RD, Kieffer TJ. Mining incretin hormone pathways for novel therapies. Trends Endocrinol. Metab. 20(6), 280-286 (2009). 
7 International Conference on Harmonization. M3(R2) Guidance on Nonclinical Safety Studies for the Conduct of Human Clinical Trials and Marketing Authorization for Pharmaceuticals. www.ich.org

8 Guerard M, Zeller A, Festag M et al. Genotoxicity testing of peptides: folate deprivation as a marker of exaggerated pharmacology. Toxicol. Appl. Pharmacol. 279(3), 419-427 (2014).

9 Heinig K, Wirz T, Schick E et al. Bioanalysis of therapeutic peptides: differentiating between total and anti-drug antibody bound drug using liquid chromatography-tandem mass spectrometry quantitation. J. Chromatogr. A. 1316, 69-77 (2013).

- Illustrates the limitation of LC-MS/MS to differentiate between active and total therapeutic peptide levels.

10 Papadimitriou A, Bansal S, Heinrich J et al. Can LC-MS/ MS and ligand-binding assays live in harmony for largemolecule bioanalysis? Bioanalysis 6(13), 1735-1737 (2014).

$11 \mathrm{Hu}$ J, Gupta S, Swanson SJ et al. A bioactive drug quantitation based approach for the detection of anti-drug neutralizing antibodies in human serum. J. Immunol. Methods 345(1-2), 70-79 (2009).

12 Wei X, Grill DS, Heatherington AC et al. Development and validation of a quantitative cell-based bioassay for comparing the pharmacokinetic profiles of two recombinant erythropoietic proteins in serum. J. Pharm. Biomed. Anal. 43(2), 666-676 (2007).

13 The Organisation for Economic Co-Operation and Development. Adopted OECD test guidelines on short, and long-term toxicity testing, with $3 \mathrm{R}$ relevance.

www.oecd.org
14 International Conference on Harmonization. Duration of Chronic Toxicity Testing in Animals (Rodent and Non Rodent Toxicity Testing). www.ich.org

15 European Medicines Agency. Guideline on bioanalytical method validation.

www.ema.europa.eu

16 Food and Drug Administration, Center for Drug Evaluation and Research, Center for Veterinary Medicine. Guidance for Industry - Bioanalytical Method Validation. www.fda.gov

17 Shankar G, Devanarayan V, Amaravadi L et al. Recommendations for the validation of immunoassays used for detection of host antibodies against biotechnology products. J. Pharm. Biomed. Anal. 48(5), 1267-1281 (2008).

18 Mire-Sluis AR, Barrett YC, Devanarayan V et al. Recommendations for the design and optimization of immunoassays used in the detection of host antibodies against biotechnology products. J. Immunol. Methods 289(1-2), 1-16 (2004).

19 Cisbio Bioassays. Product insert of cAMP dynamic 2 kit. www.cisbio.com/drug-discovery/product-insert-campdynamic-2-kit-20000-tests

20 Chiu ML, Lawi W, Snyder ST et al. Matrix effects - a challenge toward automation of molecular analysis. J. Assoc. Lab. Automat. 15, 233-242 (2010).

21 Staack RF, Jordan G, Viert M et al. Quantification of a bifunctional drug in the presence of an immune response: a ligand-binding assay specific for 'active' drug. Bioanalysis 7(24), 3097-3106 (2015). 